\title{
Tracking Excess of Maternal Deaths Directly and Indirectly Associated With COVID-19 in Brazil: A Nationwide Database Analysis
}

Raphael Mendonça Guimarães ( $\nabla$ raphael.guimaraes@fiocruz.br )

Oswaldo Cruz Foundation

Lenice Gnocchi Costa Reis

Oswaldo Cruz Foundation

Maria Auxiliadora Souza Mendes Gomes

Oswaldo Cruz Foundation

Cynthia Magluta

Oswaldo Cruz Foundation

Carlos Machado de Freitas

Oswaldo Cruz Foundation

Margareth Chrisostomo Portela

Oswaldo Cruz Foundation

\section{Research Article}

Keywords: COVID-19, SARS-CoV2, Maternal Mortality, Brazil

Posted Date: August 3rd, 2021

DOI: https://doi.org/10.21203/rs.3.rs-669001/v2

License: @ (i) This work is licensed under a Creative Commons Attribution 4.0 International License. Read Full License 


\section{Abstract \\ Background}

The COVID-19 pandemic brought a new challenge to maternal mortality in Brazil. Throughout 2020, Brazil registered 549 maternal deaths, mainly concentrated in second and third-trimester pregnant women. The objective of this study was to verify the excess of maternal deaths that occurred in Brazil caused directly and indirectly by Covid-19 in the year 2020. In addition, we sought to identify clinical social and health care factors associated with these maternal deaths, when they were directly caused by Covid- 19 .

\section{Methods}

We performed nationwide analyses, based on data from the Mortality Information System (SIM), for general deaths and maternal deaths, and the Influenza Epidemiological Surveillance System (SIVEP-Influenza), for estimates of female and maternal deaths due to COVID-19. The methodological approach adopted two distinct techniques. First, we estimated the excess maternal mortality and the breakdown of the excess between total and COVID-19 deaths. Then, we estimated the odds ratios of occurrence of symptoms, comorbidities, social determination proxies, and hospital care aspects between COVID-19 maternal and childbearing age female deaths. We chose women of childbearing age (15 to 49 years) as a reference because sex and age introduce differentials in the risk of COVID-19 death.

\section{Results}

Most of maternal deaths occurred during pregnancy in all months of $2020(\mu=59.8 \%, S D=14.3 \%$, range 50\%-100\%). Even considering the excess mortality due to COVID-19 for the childbearing age female population, maternal mortality was more penalized. Clinical variables suggest that the chances of occurrence of symptoms among maternal deaths were like those observed in the deaths of women of childbearing age. On the other hand, the odds of being a black woman, living in a rural area and being hospitalized out of the residence municipality among maternal deaths were $44 \%, 61 \%$, and $28 \%$ higher than control group. Odds of hospitalization, ICU admission, and invasive ventilatory support use among maternal deaths were, respectively, $4.37,1.73$, and 1.64 times as control group.

\section{Conclusions}

Differences between the two groups of deaths are significantly associated with social determination and (in)adequate obstetric care. Year 2021 has been more severe for maternal mortality. We need to increase immunization and monitoring among pregnant women immediately.

\section{Background}

Maternal mortality in Brazil ranged from 60 to 65 deaths per 100,000 live births between 2010 and 2017, with a considerable variation across the country [1]. As a result, Brazil did not accomplish the Millennium 
Development Goal (MDG) settled 35 deaths per 100,000 live births in 2015. In the Sustainable Development Goals (SDG) for 2030, a new compromise of 30 deaths per 100,000 live births was established. COVID-19 pandemic brought new challenges, with pregnant and postpartum women presenting a COVID-19 fatality rate of $7.2 \%$, almost three times as much as the $2.6 \%$ in the general population in the country. The group had not been identified as particularly vulnerable before when COVID-19 was still majorly affecting developed countries.

Studies have addressed the impact of COVID-19 on pregnancy, childbirth, and the puerperium and whether the pregnancy-puerperal status changes the natural history of COVID-19 [2,3]. COVID-19 infection in pregnant women has been associated with higher rates of cesarean section and mortality [4]. The effects on maternal outcomes have yet to be understood. However, we already know that the pregnant woman's risk includes relative immunodeficiency associated with maternal physiological adaptations, as well as an organic response to virus infections. Still, pregnant women can suffer from a multisystem disease, and comorbidities play a significant role in risk [5].

Maternal mortality is also certainly affected by healthcare quality, which involves access, availability of necessary resources, and acceptable practices for prenatal care, childbirth, and puerperium [6]. The impact of the pandemic on the provision of regular prenatal services and the aggravated lack of ICU beds for Obstetrics created additional difficulties in the clinical management of high-risk pregnancies, regardless of the COVID-19 infection condition [7]. Thus, we evaluated the excess of maternal deaths that occurred in 2020. After performed the decomposition of these deaths, we identified which ones were directly caused by COVID-19 and which ones could be attributed to the effects of the pandemic on healthcare services. Additionally, we verified differences between COVID-19 female and maternal deaths.

\section{Methods}

The objective of this study was to verify the excess of maternal deaths that occurred in Brazil caused directly and indirectly by Covid-19 in the year 2020. In addition, we sought to identify clinical social and health care factors associated with these maternal deaths, when they were directly caused by Covid-19.

We performed nationwide analyses, based on data from the Mortality Information System (SIM), for general deaths and maternal deaths, and the Influenza Epidemiological Surveillance System (SIVEP-Influenza), for estimates of female and maternal deaths due to COVID-19.

The methodological approach adopted two distinct techniques. First, we estimated the excess maternal mortality and the breakdown of the excess between total and COVID-19 deaths. Then, we estimated the odds ratios of occurrence of symptoms, comorbidities, social determination proxies, and hospital care aspects between COVID-19 maternal and childbearing age female deaths. We chose women of childbearing age (15 to 49 years) as a reference because sex and age introduce differentials in the risk of COVID-19 death.

\section{a) Excess Mortality}


Previously, we knew that 'x's deaths will happen in the following year. When more people die than expected, as in a pandemic, we call this discrepancy excess mortality.

The concept serves as an indicator to measure the impact of disease evolution in a population throughout the year, the emergence of others and the effectiveness of the country's health system in helping these patients. Excess mortality is calculated year by year through the difference (subtraction) between deaths from natural causes recorded between the year analyzed and the previous historical series [8]. That is, it is a difference (subtraction) between expected and recorded deaths.

In addition, we intended to verify whether the excess of maternal mortality caused by the pandemic was more significant than the deaths of pregnant women and postpartum women attributed to Covid-19 since the appearance of the first case in Brazil. That is why we describe two scenarios:

a) The number of expected maternal deaths = average maternal deaths between 2015 and 2019.

b) The number of expected maternal deaths = average maternal deaths between 2015 and 2019 plus a correction factor. We estimated this correction factor from the overall excess mortality due to COVID-19 in 2020 [9].

First, we compared the monthly count of childbearing age female deaths, maternal deaths, and COVID-19 maternal deaths in 2020 with childbearing age female and maternal deaths in the previous 5-year period (2015-2019). Then we estimated 2020 excess maternal mortality and its 95\% confidence interval using a Poisson model with robust variance. Finally, we defined two different scenarios to determine the number of excess maternal deaths: one considering the average number of maternal deaths in the previous five-year period, and another correcting that number for a factor created from the excess mortality due to COVID-19 2020 overall female mortality. We decomposed the excess maternal mortality into deaths due to COVID-19 and other causes.

\section{b) Mortality Odds Ratio}

The differential measure of risk is the basis of analytical epidemiology to describe the association between a variable of interest and a health outcome. The standardized mortality ratio (SMR) is the paradigm of these measures. It is considered a particular case of relative risk (RR). However, calculating the SMR requires information on the size of the population at risk. This information is not always available. In this situation, the most used measure is proportional mortality (PMR). However, PMR can distort the cause specific SMR measurement, mainly due to the unbalanced contribution of disease groups to the total number of deaths [10]. In this sense, other measures fulfill this role with less rigid assumptions. One of them is the Mortality Odds Ratio.

In the absence of denominator data, Miettinen and Wang [11] suggested mortality odds ratios (MORs) help obtain observed-to-expected (O/E) ratios when death data are available, but the population at risk is unknown. Wherever death certificates are the only data source used to assess risk, this is an effective measure. 
Since we used data from the Influenza Epidemiological Surveillance System (SIVEP-Influenza), filtering deaths by COVID-19, we had exactly this situation. We then applied MOR as an alternative to compare COVID-19 maternal deaths and childbearing age female deaths.

In the end, we compared female deaths and maternal deaths whose underlying cause was COVID-19. We stratified these analyses by clinical, inpatient care, and social determinants proxy variables. Given that only deaths were considered, not allowing for direct risk estimates, comparisons were performed based on mortality odds ratios (MOR) and 95\% confidence intervals.

We performed statistical analysis using R 4.0.0.

\section{Results}

In 2020, 549 COVID-19 deaths in pregnant and postpartum women were reported in the country, with a weekly average of 12.1 deaths [12]. In addition to the significant increase in deaths, it was possible to identify that most of them occurred during pregnancy and not in the puerperium in all months of $2020(\mu=59.8 \%, S D=$ $14.3 \%$, range $50 \%-100 \%$ ) with cases. Considering the first scenario for the excess maternal mortality estimation, we found a statistically significant excess mortality from March to November 2020 (Fig. 1a). In July, the peak of the first pandemic wave, there was a $79 \%$ excess of maternal deaths, compared to July in the previous five-year period. Considering the second scenario, which assumes an increase in maternal deaths due to the rise in female population mortality caused by the pandemic, we obtained a similar picture (Fig. 1b). Excess maternal mortality was still estimated between April and November. This diagnosis reinforces the idea that even with an increase in general female mortality because of COVID-19, pregnant and postpartum women were additionally penalized, with an excess of maternal mortality higher than the excess of general female mortality.

Regarding the breakdown of excess maternal deaths, we found that in 2020 most of this excess was due to COVID-19 $(\mu=70.0 \%, S D=28.2 \%$, range $6.1 \%-100 \%)$. However, an essential diagnosis was an excess of deaths that did not have to do with COVID-19 directly. Therefore, we assume that these deaths were related to barriers faced by women to timely adequate healthcare access and the worsening in the performance and quality of the care provided.

Table 1 shows the comparisons between maternal and female deaths due to COVID-19 by clinical, inpatient care and social variables using MOR (Table 1). Clinical variables suggest that the chances of occurrence of symptoms among maternal deaths were like those observed in the deaths of women of childbearing age, with no statistically significant difference. Regarding comorbidities recorded in the SIVEP-Influenza, the overall occurrence was lower among maternal deaths than among female deaths due to COVID-19 (MOR $=0.50$; $95 \% \mathrm{Cl} 0.25-0.80)$. However, the reason for this difference was due to three groups: cardiovascular diseases $(\mathrm{MOR}=0.36 ; 95 \% \mathrm{Cl} 0.09-0.66)$, diabetes $(\mathrm{MOR}=0.32 ; 95 \% \mathrm{Cl} 0,10-0.59)$ and obesity $(\mathrm{MOR}=0.53 ; 95 \% \mathrm{Cl}$ $0.21-0.85)$. The weight of these comorbidities is high due to their prevalence. The chance of their occurrence among maternal deaths was lower than in the deaths of women of childbearing age. 
Table 1

Maternal and female COVID-19 Mortality Odds Ratio (MOR) per social determinant proxy, clinical and inpatient care variables. Brazil, 2020.

\begin{tabular}{|c|c|c|c|}
\hline Dimension of Risk Factors & MOR & $\mathrm{Cl} 95 \%$ & P-value \\
\hline \multicolumn{4}{|l|}{ Social Determinant Proxies } \\
\hline Black vs Non-Black People & 1.44 & 1.22 to 1.66 & $<0.01$ \\
\hline Rural vs Urban/Periurban Place & 1.61 & 1.24 to 1.98 & $<0.01$ \\
\hline Out-of-City Hospitalization & 1.28 & 1.09 to 1.48 & $<0.01$ \\
\hline \multicolumn{4}{|l|}{ Symptoms } \\
\hline Fever & 0,91 & 0.69 to 1.14 & 0.48 \\
\hline Cough & 1.15 & 0.90 to 1.40 & 0.29 \\
\hline Throat Pain & 1.08 & 0.84 to 1.33 & 0.55 \\
\hline Dyspnea & 0.75 & 0.48 to 1.03 & 0.19 \\
\hline Respiratory Distress & 1.00 & 0.75 to 1.25 & 0.97 \\
\hline Oxygen saturation $<95 \%$ & 0.82 & 0.82 to 1.06 & 0.13 \\
\hline Diarrhea & 0.78 & 0.46 to 1.10 & 0.15 \\
\hline Vomit & 0.68 & 0.32 to 1.04 & 0.09 \\
\hline \multicolumn{4}{|l|}{ Comorbidities } \\
\hline Overall & 0.50 & 0.25 to 0.80 & $<0.01$ \\
\hline Cardiovascular Disease & 0.36 & 0.09 to 0.66 & $<0.01$ \\
\hline Hematological Diseases & 0.88 & 0.15 to 1.60 & 0.85 \\
\hline Liver Diseases & 1.33 & 0.68 to 1.99 & 0.49 \\
\hline Asthma & 0.69 & 0.21 to 1.18 & 0.16 \\
\hline Diabetes & 0.32 & 0.10 to 0.59 & $<0.01$ \\
\hline Neuropathies & 0.64 & 0.08 to 1.21 & 0.18 \\
\hline Lung diseases & 1.02 & 0.51 to 1.55 & 0.97 \\
\hline Immunodepression conditions & 0.64 & 0.25 to 1.04 & 0.08 \\
\hline Kidney Diseases & 0.61 & 0.18 to 1.03 & 0.06 \\
\hline Obesity & 0.53 & 0.21 to 0.85 & $<0.01$ \\
\hline \multicolumn{4}{|l|}{ Hospital Care } \\
\hline Hospital Admission & 4.37 & 3.39 to 5.37 & $<0.01$ \\
\hline ICU admission & 1.73 & 1.50 to 1.98 & $<0.01$ \\
\hline
\end{tabular}




\begin{tabular}{|llll|}
\hline Dimension of Risk Factors & MOR & Cl 95\% & P-value \\
\hline Invasive Respiratory Support (yes vs. no) & 1.64 & 1.42 to 1.86 & $<0.01$ \\
\hline Non-Invasive Respiratory Support (yes vs. no) & 0.57 & 0.32 to 0.82 & $<0.01$ \\
\hline
\end{tabular}

Nonetheless, the odds of being a black woman, living in a rural area and being hospitalized out of the residence municipality among maternal deaths were $44 \%, 61 \%$, and $28 \%$ higher than those in the female deaths. These findings suggest that the COVID-19 pandemic has a synergistic effect with the inequalities already observed in maternal mortality regardless of the pandemic. Finally, inpatient care variables seem to differentiate more maternal from female deaths than the clinical variables. The odds of hospitalization, ICU admission, and invasive ventilatory support use among maternal deaths were, respectively, 4.37, 1.73, and 1.64 times as much as those among female deaths. This profile suggests that pregnant and postpartum women who died were managed as critical cases.

\section{Discussion}

The result suggests that clinical characteristics did not strongly impact the excess of maternal deaths. On the other hand, variables related to hospital care and social determinants of health seem to have an apparent effect. The findings indicate that Covid-19 deaths among pregnant and postpartum women, compared to childbearing age females, are not attributable to other clinical aspects such as comorbidities than the pregnancy itself. Therefore, once there is no stronger than marginal association to comorbidities, we can consider pregnancy an independent risk factor of dying from Covid-19. It requires attention to establish actions to reduce this risk. This situation gets worse when we consider all the problems due to access to health services from those women, which can explain why Brazil keeps the rates at this level.

Since mid-2020, publications on the deaths of pregnant and postpartum women due to Covid-19 in Brazil have alerted to the need to prepare and organize the complete healthcare services network. They focused on ensuring timely access and the adequacy of clinical practices because of the specificities of the disease in the pregnancy-puerperal cycle. Brazil has a maternal mortality ratio compared to countries with average sociodemographic development, according to a composed index of per capita income, average education, and fertility. Given that Brazilian fertility is at a lower level, the social and income inequalities are the elements that explain Brazil's position. High maternal mortality rates are mainly concentrated in countries with a peripheral economy and reveal a severe violation of the right to health. Maternal mortality is a preventable event in more than $90 \%$ of cases. It is a determining factor in the increase in poverty and starvation [13].

The more outstanding picture of social inequality plays the central role [14]. Differences among pregnant women's profiles in Brazil and other countries are not unlikely. There is a high occurrence of comorbidities in the country whose etiology involves inflammatory conditions, which are risk factors for complications from Covid-19, such as obesity, hypertension, diabetes and vasculopathies [15]. Social determinants of health strongly influence these chronic conditions. Racial, geographic, and socioeconomic disparities, therefore, require special attention. Social, economic and health policies would be benefitted from consideration of this contextual effect [16]. 
In developing, as opposed to developed countries, high birth rates and limited resources for healthcare delivery contribute to the increased risk of maternal death due to COVID-19 [17]. Brazil, however, has had a declining birth rate since the 1970s, and poor quality prenatal and obstetrical care have a more powerful explanatory weight. Timely access to adequate obstetric services is essential for patient safety and quality of maternal care [18]. Delay in receiving it is associated with the severity of adverse maternal outcomes. Thadeus \& Maine [19] developed a model called "Three Delays" to assess access to obstetric care, broken down into three components or phases: (1) delay in the decision to seek health care; (2) delay in identifying and reaching the appropriate health service; and (3) delay in receiving appropriate care at the right time. Even today, this model is used to explain most maternal deaths and some cases of severe maternal morbidity.

In Brazil, barriers to access obstetric services with specialized complex care, and inadequate monitoring of obstetric complications, persist [20]. It occurs despite the warning that the CDC has issued about COVID-19 increased risk for pregnant and postpartum women to develop into severe forms, requiring hospitalization, intensive care, use of mechanical ventilation, and even premature births [21]. In addition to the impressive number of 2020, over less than six months of 2021, maternal deaths had already surpassed the number reported in the previous year. There are 1442 deaths registered. Of all maternal deaths, $848(58.9 \%)$ are pregnant women, and 594 (41.2\%) are postpartum women. It represents more than twice of the previous year [22].

For all these reasons, the COVID-19 pandemic may represent a significant immediate obstacle to Brazils' achievement of SDG by 2030. The excess of maternal mortality and the significant increase in near-miss cases caused by COVID-19, directly or indirectly, put the country in a delicate situation.

Our analysis has limitations. Total mortality data represent data available at the time of research. We recognize that the data can change due to some updates over the next few months. However, even if there is a recovery of maternal death records for the database, maternal deaths pattern is already worrying in the current scenario. Another limitation concerns the low testing performed in Brazil. This limitation prevents us from knowing precisely how many COVID-19 infected pregnant and postpartum women. However, this information does not compromise the estimate calculation we use. The Mortality Odds Ratio has the advantage that we can use it precisely in this type of circumstance, where the population base is unknown. We assume deaths in the general population (in our case, the childbearing age female population) to estimate the odds of factors we considered related to maternal mortality. We also know that there is some imbalance between the age group that concentrates most women who experience pregnancies (between 20 and 29 years old) and the group with the highest prevalence of comorbidities (40 to 49 years old). However, we performed the analyses disregarding pregnancies' order, and we believe this minimizes the potential selection bias.

The situation in Brazil demonstrates the importance of national leadership in confronting the pandemic. It is even more important to recognize the need for long-term global care to improve local public health [23]. High maternal mortality is an indicator of failure in the organization of health services. Moreover, the solution requires the involvement of the international community since it affects global development. This scenario does not repeat itself in other low- and middle-income countries, prolonging the pandemic's impact for all [24]. For this reason, the analysis of excess maternal mortality is a call of action at this point in the pandemic. 
That said, we emphasize the need for adequate measures for adequate prenatal, delivery and postnatal care. It is fundamental to prepare the healthcare network so that pregnant and postpartum women do not remain unattended, further aggravating maternal mortality.

We assume that the vaccination performed on pregnant women in the first trimester is adequate to protect pregnant women during the second and third trimesters of pregnancy. Moreover, it is necessary to combine non-pharmacological measures and vaccination. We need to strengthen maternal health care, from access to prenatal care to the regulation of obstetric ICU beds. Prenatal consultations must be qualified, encouraging physical distance measures. We also need to screen pregnant women for respiratory symptoms, distribute good quality masks, adopt universal testing on admission to maternity hospitals with molecular tests (RTPCR), and provide obstetric care in hospital units with access to ICU beds for moderate cases and ICU beds for severe cases.

\section{Declarations}

\section{Ethics approval and consent to participate}

Not applicable

\section{Consent for publication}

Not applicable

\section{Availability of data and materials}

The datasets generated and/or analysed during the current study are available in the Ministry of Health of Brazil,SRAG 2020 - Severe Acute Respiratory Syndrome Database - including data from COVID-19 -repository, available at https://opendatasus.saude.gov.br/dataset/bd-srag-2020

\section{Competing interests}

The authors declare that they have no competing interests

\section{Funding}

Not applicable

\section{Authors' contributions}

RMG, LCR, MASMG and CM conceived the research. RMG and MCP defined the methodology. RMG and MCP conducted the formal analysis. RMG, MCP, MASMG and CM interpreted the data. RMG produced all visualization. RMG and MCP wrote the first draft of the manuscript. All authors contributed to the interpretation of results and manuscript editing.

\section{Acknowledgements}

Not applicable 


\section{Authors' information}

The researchers are part of the Covid-19 Fiocruz Observatory. The Oswaldo Cruz Foundation is the largest public health center in Latin America, and since the beginning of the pandemic, it has been continuously monitoring pandemic indicators in Brazil, describing epidemiological scenarios and identifying groups or situations of greater vulnerability to the progress of Covid-19 in the country.

\section{References}

1. WHO, UNICEF, UNFPA. UNICEF Data Warehouse: Maternal mortality ratio, Brazil. Available at https://data.unicef.org/resources/data_explorer/unicef_f/? $\mathrm{ag}=\mathrm{UNICEF} \& \mathrm{df}=\mathrm{GLOBAL} \_D A T A F L O W \& \mathrm{ver}=1.0 \& \mathrm{dq}=\mathrm{BRA} . \mathrm{MNCH} \_M M R . \&$ startPeriod=1970\&endPeriod=2021

2. Takemoto, M.L.S., Menezes, M.O., Andreucci, C.B., Knobel, R., Sousa, L.A.R., Katz, L. et al. Maternal mortality and COVID-19. J Matern Fetal Neonatal Med. 16, 1-7 (2020).

3. Nakamura-Pereira, M., Amorim, M.M.R., Pacagnella, R.C., Takemoto, M.L.S., Penso, F.C.C., Rezende-Filho, J. et al. COVID-19 and Maternal Death in Brazil: An Invisible Tragedy. Rev Bras Ginecol Obstet. 42(8),445447 (2020).

4. Karimi, L., Makvandi, S., Vahedian-Azimi, A., Sathyapalan, T., Sahebkar, A. Effect of COVID-19 on Mortality of Pregnant and Postpartum Women: A Systematic Review and Meta-Analysis. J Pregnancy. 8870129 (2021).

5. Cimolai, N. A Comprehensive Analysis of Maternal and Newborn Disease and Related Control for COVID19. SN Compr Clin Med. 17, 1-23 (2021).

6. Lumbreras-Marquez, M.I., Campos-Zamora, M., Lizaola-Diaz de Leon, H., Farber, M.K. Maternal mortality from COVID-19 in Mexico. Int J Gynaecol Obstet. 150(2), 266-267 (2020).

7. Souza, A.S.R., Amorim, M.M.R. Maternal mortality by COVID-19 in Brazil. Revista Brasileira de Saúde Materno Infantil. 21(Suppl 1), 253-256 (2021).

8. Green, H.K., Andrews, N.J., Bickler, G., Pebody, R.G. Rapid estimation of excess mortality: nowcasting during the heatwave alert in England and Wales in June 2011. J Epidemiol Community Health. 66, 866-8 (2012).

9. Silva, G.A., Jardim, B.C., Santos, C.V.B.D. Excess mortality in Brazil in times of Covid-19. Cien Saude Colet. 25(9), 3345-3354 (2020)

10. Stewart, W., Hunting, K. Mortality odds ratio, proportionate mortality ratio, and healthy worker effect. Am J Ind Med. 14(3), 345-53 (1998).

11. Miettinen, O.S., Wang, J.D. An alternative to the proportionate mortality ratio. Am J Epidemiol. 114(1):1448 (1981).

12. Nakamura-Pereira, M., Knobel, R., Menezes, M.O., Andreucci, C.B., Takemoto, M.L.S. The impact of the COVID-19 pandemic on maternal mortality in Brazil: 523 maternal deaths by acute respiratory distress syndrome potentially associated with SARS-CoV-2. Int J Gynaecol Obstet. 153(2), 360-362 (2021).

13. Subbaraman, N. Pregnancy and COVID: what the data say. Nature. 591(7849), 193-195 (2021). 
14. Islam, M.M. Social Determinants of Health and Related Inequalities: Confusion and Implications. Front Public Health. 7, 11 (2019).

15. Yee, J., Kim, W., Han, J.M., Yoon, H.Y., Lee, N., Lee, K.E. et al. Clinical manifestations and perinatal outcomes of pregnant women with COVID-19: a systematic review and meta-analysis. Sci Rep. 10(1), 18126 (2020).

16. Gurzenda, S., Castro, M.C. COVID-19 poses alarming pregnancy and postpartum mortality risk in Brazil. EClinicalMedicine. 36, 100917 (2021).

17. Amorim, M.M.R, Takemoto, M.L.S., Fonseca, E.B.D. Maternal deaths with coronavirus disease 2019: a different outcome from low- to middle-resource countries? Am J Obstet Gynecol. 223(2), 298-299 (2020).

18. Pacagnella, R.C., Cecatto, J.G., Parpinelli, M.A., Souza, M.H., Haddad, S.M., Costa, M.L., et al. Delays in receiving obstetric care and poor maternal outcomes: results from a national multicentre cross-sectional study. BMC Pregnancy Childbirth. 14, 159 (2014).

19. Thaddeus, S., Maine, D. Too far to walk: maternal mortality in context. Soc Sci Med. 38, 1091-110 (1994).

20. Bittencourt, S.D., Domingues, R.M., Reis, L.G., Ramos, M.M., Leal, M.D. Adequacy of public maternal care services in Brazil. Reprod Health. 13 (Suppl. 03), 120 (2016).

21. Pountoukidou, A., Potamiti-Komi, M., Sarri, V., Papapanou, M., Routs,i E., Tsiatsiani, A.M. et al. Management and Prevention of COVID-19 in Pregnancy and Pandemic Obstetric Care: A Review of Current Practices. Healthcare (Basel). 9(4), 467 (2021).

22. Fiocruz' COVID-19 Observatory. Extraordinary Report - 500,000 deaths in Brazil Available at https://agencia.fiocruz.br/sites/agencia.fiocruz.br/files/u34/boletim_extraordinario_2021-junho-23parte2-pags09-17.pdf

23. Hallal, P.C., Victora, C.G. Overcoming Brazil's monumental COVID-19 failure: an urgent call to action. Nat Med. 27(6),933 (2021).

24. To curb COVID-19, global health must go local. Nat Med. 27(6),929 (2021).

\section{Figures}




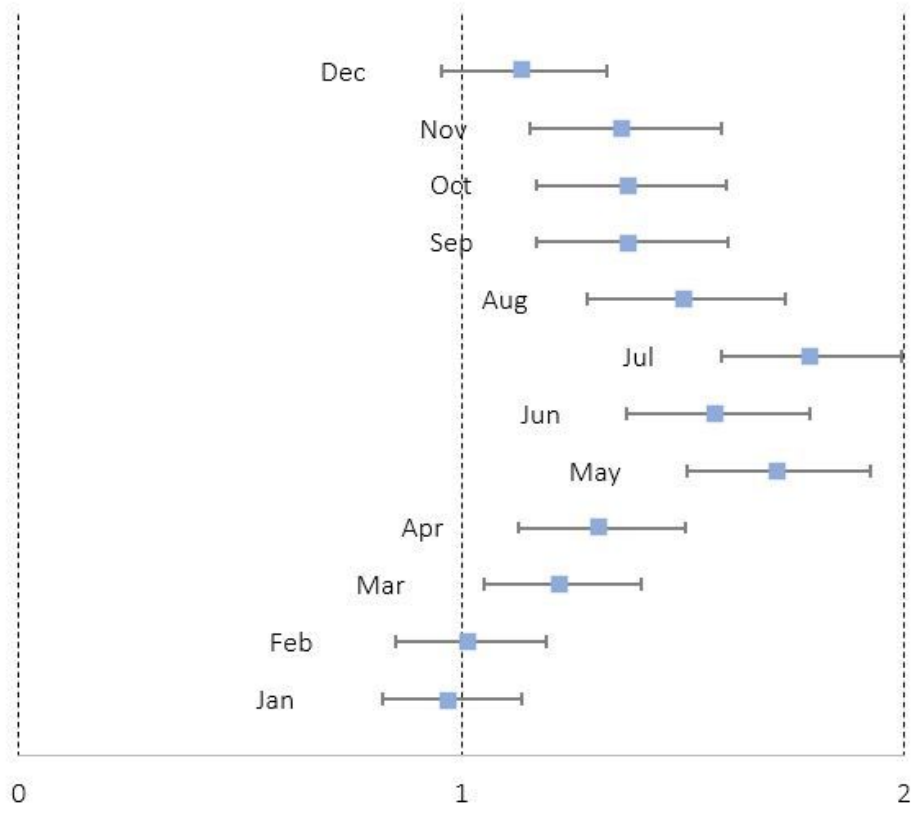

(a)

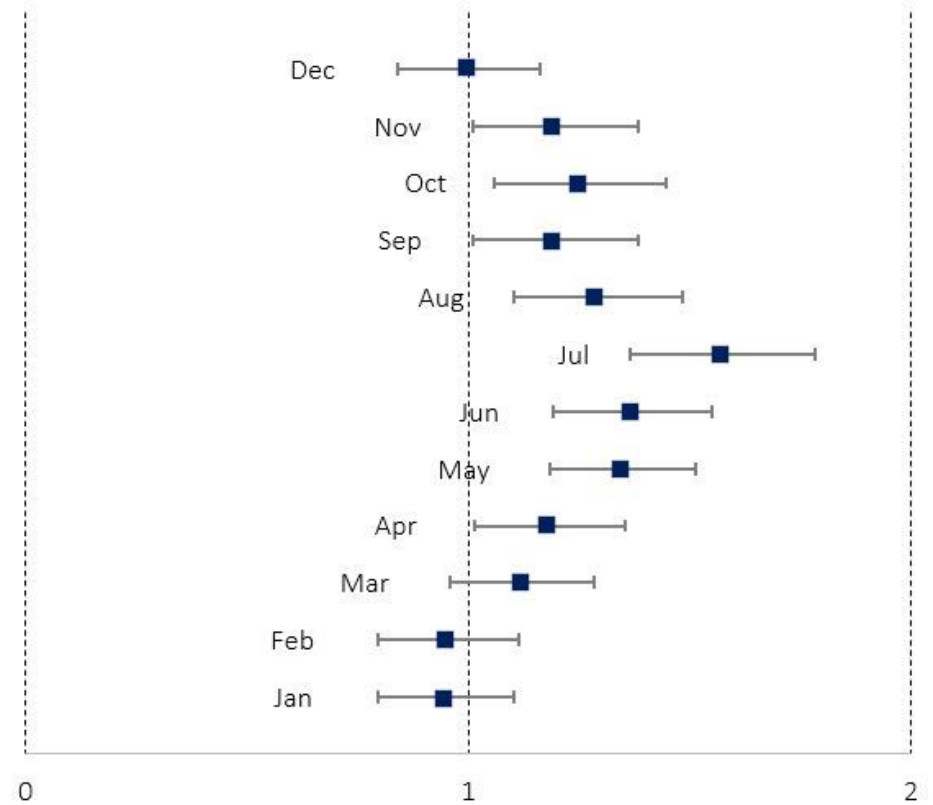

(b)

\section{Figure 1}

Excess maternal mortality per calendar month according to expected death scenarios. Brazil, 2020. Source: SIVEP-Gripe, 2021. 